\title{
Cómo diseñar una investigación para el análisis de las relaciones de género. Aportaciones metodológicas*
}

\author{
Carmen Botía-Morillas \\ Universidad Pablo de Olavide (UPO). Departamento de Sociología \\ carmenbotia@upo.es
}

\section{Resumen}

El artículo muestra el modo de aplicación del método cualitativo en la práctica, específicamente, las decisiones metodológicas tomadas para una investigación cuyo objetivo es conocer cómo se están transformando las relaciones de género. Asimismo, se especifican los principios teóricos que fundamentan estas decisiones. El método cualitativo y la técnica de investigación de la entrevista abierta se juzgan como los más adecuados para el anterior objeto de investigación, ya que inciden tanto en los esquemas simbólicos de los sujetos sociales que interaccionan, como en los procesos objetivos y prácticos que enmarcan estos procesos de interacción dentro de las relaciones cotidianas. Se apuesta y se fundamenta la importancia de seguir un diseño muestral homogéneo. Se ha entrevistado a mujeres y varones de parejas heterosexuales no tradicionales, por separado y en dos momentos de su ciclo vital: durante su primer embarazo y cuando el bebé tenía aproximadamente un año de vida. Se cuenta, por tanto, con cuatro entrevistas abiertas para cada pareja, calidad y cantidad de información con enormes potencialidades para el análisis de un objeto de investigación dinámico. Este enfoque metodológico permite conocer las contradicciones y las diferentes legitimidades en torno a los cuidados en parejas que tratan de ser igualitarias y a las que la realidad de los contextos sociales en los que interaccionan puede dificultarles el mantenimiento de relaciones de género más equilibradas. Para concluir, el artículo argumenta la validez y la calidad de la investigación cualitativa longitudinal llevada a cabo. Palabras clave: investigación cualitativa; entrevista abierta; validez; igualdad de género; nueva maternidad; nueva paternidad.

* Agradezco a la profesora Carmuca Gómez Bueno y a Alejandro D. Duarte Sánchez las observaciones, comentarios y revisión de este artículo. El artículo presenta la metodología seguida en la tesis doctoral de la autora, dirigida por el Dr. D. Enrique Martín Criado (UPO) y tutorizada por la Dra. Ma Luisa Jiménez Rodrigo (US). He contado con financiación del proyecto I+D+I del Instituto de la Mujer del Ministerio de Trabajo y Asuntos Sociales de España: 033/07 «Influencia de los valores familiares en la definición de las estrategias de distribución del tiempo en los hogares españoles», dirigido por la Dra. Mercedes Camarero Rioja (UPO). 
Abstract. How to design research for analyzing gender relations. Methodological contributions

This article explains how to apply the qualitative method in practice. Specifically, it focuses on the methodological decisions to be taken when conducting research on changing gender relations and the theoretical principles that support these decisions. The qualitative research method and the open-interview technique are considered the most adequate ways to achieve the research objective since both focus on the symbolic systems of social subjects in interaction and on the objective and practical processes that shape the framework of daily interactions. The article supports and emphasizes the importance of a homogeneous sample design. Interviews of women and men forming non-traditional heterosexual partnerships were conducted separately at two stages of their life cycle: during pregnancy and when the child was one year old. Four open interviews were made of each couple, thus providing a large amount of quality information with enormous potential for the analysis of a dynamic research object. This methodological approach provides deeper knowledge of the contradictions and conditions of legitimacy surrounding caring practices in couples that attempt to be egalitarian, but whose social interactions hinder more balanced gender relations. The article concludes with an argument on the validity and quality of the longitudinal qualitative research carried out.

Keywords: qualitative research; open interview; validity; gender equality; new motherhood; new fatherhood.

\section{Sumario}

1. Introducción, problema 6. Cómo un cuidado trabajo de campo

y objeto de investigación vela por la calidad de la investigación

2. Por qué el método cualitativo de investigación es el que mejor 7. Cómo el proceso de análisis contribuye se adecua al objeto de análisis

3. Por qué la entrevista abierta como técnica de investigación a la validez

8. Debatiendo en torno a la calidad y a la validez de las propuestas metodológicas presentadas

4. Por qué un diseño muestral homogéneo

5. Por qué entrevistar por separado y no conjuntamente

9. Conclusiones y discusión

Referencias bibliográficas

Anexo 1

\section{Introducción, problema y objeto de investigación}

El artículo presenta la metodología seguida en una investigación cuyo objetivo es explicar las condiciones de posibilidad y las dinámicas sociales que favorecen o dificultan una mayor igualdad entre géneros. Se analizan dos momentos en el tiempo que marcan la historia de una pareja: uno, el embarazo y, otro, el año posterior al nacimiento de su primer bebé, un momento especialmente demandante de tiempo de cuidados. A pesar de que, en las últimas décadas, la igualdad de género se ha convertido en el discurso legítimo en muchos países occidentales, es necesario observar qué sucede con las dinámicas sociales y con las relaciones entre varones y mujeres en sus contextos cotidianos. Las parejas 
analizadas se identifican como igualitarias durante el embarazo, pero, tras el nacimiento de su primer bebé, les resulta más complicado mantener estas relaciones de igualdad. He tratado de conocer si realmente está habiendo un cambio y si las relaciones de género están pasando de ser más tradicionales a más equilibradas, tanto en el ámbito público con relación al empleo, como en el ámbito privado o el del hogar. Esta investigación explora tanto los procesos subjetivos o esquemas simbólicos de los sujetos sociales que interaccionan, como las estructuras o los procesos objetivos y prácticos que enmarcan los procesos de interacción que tienen lugar en las relaciones cotidianas, para así poder conocer las contradicciones y las transformaciones que se están produciendo.

El artículo parte de una realidad presente en las estadísticas que muestran que hay diferencias en las prácticas de cuidado de cada miembro de la pareja en función de su género, especialmente cuando se convierten en madres y padres, a pesar de haber sido parejas más igualitarias antes de la llegada del primer bebé. Generalmente, el aumento de necesidades temporales conlleva una vuelta a formas tradicionales de relación (Weismann et al., 2008). Además, la carga temporal que requieren los cuidados disminuye a medida que aumenta la edad de los hijos (Meil, 1997), por lo que aquella es mayor cuanto más pequeños son estos. Realmente, para una madre, las diferencias fundamentales se establecen entre tener o no tener hijos, además, cuanto más grande es la familia y más pequeños los hijos, mayor segregación y carga de trabajo tendrán las madres respecto a los padres (Regnier-Loilier, 2009).

El objetivo de la investigación cuya metodología se presenta aquí es analizar las relaciones de género, un objeto de investigación dinámico e interactivo. Aporto un enfoque propio que examina la posibilidad de transformación de las relaciones de género abordando las estrategias y las prácticas de negociación puestas en juego para resolver conflictos entre géneros mediante la movilización de distintos recursos con valor para la pareja, ya sean estos económicos, simbólicos, de tiempo, educativos u otros posibles; tangibles o intangibles. El enfoque teórico de la investigación se basa en el concepto de poder de Elias (1982), entendido como poder interrelacional y recíproco y, por tanto, elemento integral que constituye todas las relaciones sociales, que pueden ser entendidas como interdependientes, no cerradas ni fijas, sino con posibilidad de ser modificadas. Esta acepción de poder es fundamental para analizar la posibilidad de transformación de las relaciones de género (Botía, 2010). Las interacciones de una pareja en sus contextos cotidianos plantean tensiones clave para identificar las condiciones de posibilidad, los procesos y las dinámicas sociales que les afectan y que contribuyen a generar. Estas pueden dificultar o facilitar el equilibrio en las relaciones de género durante la convivencia y se derivan, entre otras, de las exigencias del empleo remunerado de mujeres y varones, de la implicación de los padres en los cuidados, de la red de ayuda familiar o de la delegación, por parte de las madres, de las responsabilidades de cuidados.

Hay muchas perspectivas teóricas que explican la división del trabajo por razón de género. Sin poder mencionarlas todas, estaría el enfoque que considera que el género tiene suficiente entidad como para afectar a diferentes niveles 
de la sociedad (Risman, 1998 y 2004; Connell, 1987), así, por ejemplo, en el plano personal, influye en las diferentes expectativas de varones y mujeres; asimismo, los recursos institucionales o materiales se distribuyen desigualmente en función del género. La consideración del género como un factor estructural permite explicar un conjunto complejo de valores, expectativas, responsabilidades y roles que influye en la distribución diferente de recursos, en las diferentes oportunidades institucionales o en las diferencias ideológicas.

En el plano individual, y con relación a las interacciones de pareja, la economía y la sociología han avanzado teorías sobre la participación de los hombres en el trabajo no remunerado. La primera, Becker (1981), se centra en los recursos económicos y de tiempo, enfoque que no incorpora un análisis crítico sobre la distribución desigual de recursos entre varones y mujeres, puesto que ellas son consideradas «más adecuadas» para desempeñar el trabajo no remunerado, por su posición subordinada. Más tarde, desde la teoría feminista y desde las teorías de intercambio económico, se plantea la división del trabajo como una situación en la que ambos miembros de la pareja tienen conflictos de intereses y recursos relativos, y no están de acuerdo con que el marido y la mujer compartan las mismas preferencias y tomen decisiones conjuntamente, como planteaba Becker (Manser y Brown, 1980), ya que el titular del salario tiene más posibilidades de negociar dentro y fuera del hogar. Es más posible negociar intereses propios cuanto más recursos se manejan y, por eso, la centralidad del empleo de las mujeres o el aumento de su nivel educativo, entendidos como recursos, pueden facilitarlo. Asimismo, que los padres tengan un empleo que les deje tiempo disponible puede facilitar su dedicación a los cuidados familiares (Dermott, 2006 y 2008). No obstante, es necesario incorporar un análisis de género, entendido como ideología que construye diferencias entre varones y mujeres previamente al análisis de los recursos que se manejan, ya que puede sobrevalorarse la contribución de los varones a la relación, con lo cual se podrían satisfacer en mayor medida sus intereses y no los de las mujeres, aunque sean estas quienes contribuyan con mayores ingresos a los recursos económicos conjuntos (Dema, 2005 y 2006). De hecho, puede suceder que las mujeres no sean siquiera conscientes de los recursos propios que ponen en juego en su relación de pareja y, por tanto, no puedan identificarlos para posicionarse mejor en sus negociaciones cotidianas (Coria, 1996).

Esta realidad desigual en función del género puede explicarse por la forma como se construyen las relaciones entre varones y mujeres y como ambos miembros de la pareja se socializan conformándose a diferentes roles, expectativas e intereses familiares y con relación al trabajo. Así, la ideología de género contribuye a generar diferentes recursos en función de si se es varón o mujer (Zuo y Bian, 2001). También la socialización de género explica las diferentes preferencias de varones y mujeres, que hacen que éstas opten en mayor medida por asumir el trabajo doméstico y de cuidados (Hakim, 2000). El género como elemento estructural explica también los diferentes estándares de las mujeres respecto a espacios considerados tradicionalmente masculinos. En el empleo, por ejemplo, los estándares de comparación son diferentes para varones y 
mujeres. Así, las mujeres, en lugar de compararse con los varones, se sienten afortunadas al compararse con otras mujeres, sin percibir las discriminaciones a las que se enfrentan continuamente, que serían visibles si la referencia de comparación fuesen los varones (Babcock y Laschever, 2003).

Esta realidad desigual construida por las relaciones de género influye en varones y mujeres, que terminan comportándose como se espera en relación con su género, con lo cual se reproducen las relaciones desiguales entre ambos miembros de la pareja. El análisis de la reproducción social por razón de género se ubica en los llamados «estudios doing gender», sin embargo, el estudio cuya metodología se presenta aquí trata de identificar los elementos presentes en parejas reales que cuestionan estos mandatos y estas relaciones tradicionales de género, por ello se enmarcaría en los «estudios undoing gender». Además, los contextos sociales en los que vive y se desarrolla cada relación de pareja pueden constituir oportunidades y obstáculos para llevar a cabo una relación menos tradicional. Esto es precisamente lo que se investiga, la forma como parejas concretas, seleccionadas teóricamente por su mayor posibilidad de tener un equilibrio de género que parejas tradicionales, logran, o no, seguir una práctica de mayor igualdad.

\section{Por qué el método cualitativo de investigación es el que mejor se adecua al objeto de análisis}

Son numerosas las investigaciones cuantitativas que evidencian las diferencias de tiempo que varones y mujeres dedican a realizar tareas domésticas y a cuidar de los suyos (Durán y Rogero, 2009), lo cual muestra la vivencia de unas relaciones de pareja no igualitarias; o las diferencias en el tiempo utilizado por los padres para el permiso de paternidad frente al utilizado por las madres para el de maternidad, punto de partida de una implicación diferencial posterior de padres y madres a los cuidados (Meil, 2011). En otras investigaciones, pueden observarse las diferentes responsabilidades asumidas y las expectativas esperadas para varones y mujeres que conviven (Durán, 1978; Hartmann, 1981; Segalen, 1992; Brullet, 1996; Meil, 1997; Carrasco, 2003), diferencias de género que se acrecientan tras el nacimiento del primer hijo (Esping-Andersen y Brodmann, 2005; Regnier-Loilier, 2009).

Complementariamente a estos estudios y publicaciones que ponen de manifiesto la existencia de una realidad social desigual para varones y mujeres, la estrategia cualitativa se torna fundamental para comprender la forma como, en la práctica, se asumen estas diferencias, reproduciéndolas o tratando de superarlas. Bott (1990) o Miller (2007 y 2011), ambas con un objetivo comprensivo sobre las relaciones de pareja, utilizan la estrategia cualitativa. Un estudio holandés que trata de entender las dinámicas cotidianas y la toma de decisiones en parejas con hijos también recomienda el método cualitativo: "Qualitative in depth research has therefore proved indispensable in gaining a better understanding of the daily dinamics of decisions-making in couple» (Weismann et al., 2008: 359). Del mismo modo, Soledad Murillo (1996) 
considera el abordaje cualitativo fundamental para conocer cómo tienen lugar las diferencias sociales, y específicamente de género, en los distintos espacios colectivos. También Teresa Torns (2008) argumenta la necesidad de llevar a cabo estudios cualitativos para analizar el concepto de trabajo, el trabajo doméstico y los cuidados, ámbitos objeto de la investigación cuya estrategia metodológica presento aquí.

Así, la identificación, la explicación y, especialmente, la comprensión de las dinámicas que llevan a relaciones más igualitarias entre varones y mujeres demandan una aproximación metodológica cualitativa. Explicar pormenorizadamente la forma como se debe proceder es la apuesta del presente artículo. La estrategia cualitativa es más capaz de detectar lo relevante y se interesa por el sentido que los sujetos dan a sus acciones, es interpretativa, integradora y abierta a la voz de quienes son investigados; sin olvidar que, en el método cualitativo, el lenguaje es fundamental, es el objeto propio de estudio, pudiendo tratar los casos investigados de manera intensiva (Beltrán, 1998). El enfoque cualitativo, además de posibilitar la intersubjetividad y la interpretación, permite abordar las interacciones que se establecen en los espacios sociales concretos, atendiendo tanto a las estructuras y a las instituciones como a la percepción de las relaciones en la vida social. Es la aproximación metodológica que permite centrarse en la vida cotidiana y en sus dinámicas e interacciones (Alonso, 1998).

De hecho, el método cualitativo permite dar sentido a la realidad, integrada por comportamientos consensuados y conflictivos, por estrategias de interacción y negociación en la vida cotidiana, especialmente en torno al empleo, los cuidados y el uso de los tiempos. He tratado de conocer cómo las prácticas o las estrategias de interacción pueden modificar los «equilibrios de poder ${ }^{1}$ » (Elias, 1982), y analizar si es posible el cambio de relaciones de género para constituirse en más igualitarias a partir de prácticas concretas y de la movilización de los recursos de mujeres y varones. Al mismo tiempo, he tratado de explicar cómo realmente están funcionando estas estrategias en los contextos sociales cotidianos.

\section{Por qué la entrevista abierta como técnica de investigación}

La técnica de investigación que se ha considerado más adecuada ha sido la entrevista abierta, ya que interesaba conocer cómo mujeres y varones explican sus interacciones y negociaciones cotidianas con su pareja; los roles y funciones que desempeñan; incluso los temas o las situaciones implícitas asumidas y los que generan conflicto o malestar. La entrevista abierta se caracteriza por

1. Para Norbert Elias (1982), «los equilibrios de poder» pueden entenderse como situaciones en las que existe una determinada relación de interdependencia y reciprocidad en un momento y en un contexto social concreto, con lo cual es posible que estos equilibrios fluctúen. Que exista reciprocidad no significa que haya equivalencia en los recursos y en la influencia que pueden ejercerse quienes interaccionan. 
su apertura, flexibilidad y dinamismo, así como por su potencialidad para ofrecer testimonios personales que son interesantes, en tanto que constituyen una expresión biográfica de lo social. Es una técnica que responde al objetivo de aprender, y no tanto al de extraer respuestas de los sujetos sociales (Taylor y Bogdan, 2002), algo fundamental para esta investigación. He tomado los relatos como prácticas en sí mismas, lo que constituye el valor que las entrevistas aportan, y ello me ha permitido conocer a las personas lo suficiente como para comprender cómo se ven a sí mismas, a su mundo (Taylor y Bogdan, 2002) y al objeto de investigación. Es necesario, además, que la investigación sea longitudinal, debido a que el objeto que se analiza es dinámico y está en proceso de transformación continuo.

Es innovador que el carácter longitudinal se aplique a estudios cualitativos que investigan los procesos sociales en el momento en que se están produciendo. Así, la investigación ha partido del momento en el que se anticipan los cuidados, durante el embarazo y han llegado hasta un año después del nacimiento, cuando ya se han puesto en juego las prácticas y las experiencias de cuidados. La entrevista abierta es una técnica que posibilita este abordaje.

Cada entrevista ha constituido un proceso comunicativo y de relación personal establecido en el contexto concreto en el que se ha desarrollado y en el que se han generado los discursos finalmente analizados. Las entrevistas no se han considerado como textos lineales, ya que la comunicación cotidiana no es lineal, sino como conjuntos contextuales de los sujetos, como conjuntos de actos de habla, como un todo, como narraciones globales. Así, cada entrevista ofrece una buena información, tanto del sujeto social que se expresa en ella, como del contexto social, y concretamente de género, que configura las condiciones de existencia de cada persona. La selección y contactación de las parejas entrevistadas han sido procesos fundamentales para garantizar la validez de la investigación, más adelante se explica la forma como se ha procedido en estas fases.

En las entrevistas he priorizado el análisis de los aspectos estructurales, sociales y comunes al contexto de análisis, más que los emocionales y personales. Asimismo, he tratado de controlar los acuerdos previos al comienzo de cada entrevista mediante quienes han contactado con las parejas y al inicio de cada una de ellas, a fin de evitar poner en riesgo la veracidad de la información generada en las entrevistas y, por tanto, la validez de la investigación.

Ha sido el objeto de investigación el que ha demandado la entrevista abierta como la técnica de investigación más adecuada. He descartado otras técnicas, como la observación participante, ya que habría que haber estado presente en la vida de cada pareja durante el primer año tras el nacimiento de su bebé, además de en momentos extensos durante la gestación, lo que habría supuesto un trabajo de campo inviable en la práctica. Del mismo modo, he descartado el grupo de discusión, ya que no se trataba de conseguir un discurso legítimo sobre cómo ser buen padre o buena madre, cómo cuidar a la pareja o cómo negociar con ella; ni de reconstituir el sentido que un colectivo da a un fenómeno social, ni de reconstruir un significado conjunto (Martín Criado, 1997) o representativo de la posición social del grupo (Conde, 2008). También he 
descartado la técnica del grupo triangular, ya que tampoco se trata de analizar el proceso de construcción discursiva en la interacción que se genera en un espacio social; como tampoco de incorporar discursivamente algo que viene de fuera, ni de proyectar hacia fuera algo de dentro (Conde, 2008), sino que de lo que se trataba era de llegar a las contradicciones presentes en las prácticas diarias y conocer las dificultades para alejarse de roles y responsabilidades tradicionales por razón de género cuando llega un bebé a una pareja. Para ello, he partido de la propia experiencia y trayectoria personal de cada sujeto, incorporando su punto de vista. Sin duda, la técnica que mejor puede abordarlo es la entrevista abierta.

\section{Por qué un diseño muestral homogéneo}

La explicación de la selección muestral es relevante, ya que está basada en fundamentos teóricos que explican la posibilidad de vivir dentro de una relación que permita una mayor igualdad entre géneros que la que se daba en parejas tradicionales. He seguido, por tanto, un muestreo teórico. Han sido trece las parejas entrevistadas en dos momentos y por separado, salvo tres de ellas, entrevistadas conjuntamente. Las primeras entrevistas a cada pareja se han realizado durante el periodo de gestación y las segundas, cuando el bebé tenía aproximadamente un año. Para identificar los aspectos más relevantes en relación con el objetivo de investigación y poder establecer relaciones teóricas es necesario que exista poca variación entre los casos entrevistados, esto es posible con la homogeneidad muestral (Pole y Lampard, 2002: 37, basado en Patton, 1990, y Glaser y Strauss, 1967). Los estudios cuantitativos, por el contrario, basan su validez fundamentalmente en la heterogeneidad muestral y en la amplitud de sus muestras, pero un diseño cualitativo necesita la homogeneidad para hacer extensibles sus resultados a contextos como el analizado. Por tanto, el proceso teórico de muestreo adoptado ha perseguido la homogeneidad, para poder ofrecer explicaciones sobre las dinámicas sociales que apoyan y dificultan la igualdad de género en parejas alejadas del modelo tradicional de roles segregados que transitan hacia un modelo de mayor equilibrio de género.

Cada persona entrevistada, y cada pareja, ha sido considerada como un sujeto social no intercambiable ni ajeno a la realidad social que lo constituye, ya que es su posición social lo que le avala para formar parte de la muestra. Téngase en cuenta que no se investigan en tanto sujetos sociales concretos, sino en tanto agentes en los que poder analizar las relaciones de género en la práctica. El proceso de muestreo y la selección de cada pareja entrevistada ha atendido a los siguientes criterios teóricos, que son relevantes y se justifican en función de lo que podían ofrecer potencialmente para la investigación:

a) Parejas que manifiestan y están de acuerdo con la corresponsabilidad en el cuidado de su primer bebé y en la realización de tareas o actividades de cuidados y gestión del hogar. Esta característica se identifica por el conocimiento sobre la forma de interaccionar y asumir las tareas en el empleo y el trabajo no 
remunerado durante el embarazo, considerado el punto de partida mínimo para alejarse de roles tradicionales.

b) Ambos miembros de la pareja con empleo a tiempo completo. El elemento que más iguala a la pareja es su papel productor fuera del hogar (Carabaña, 1993). La realidad laboral general para las mujeres, más precaria, ha llevado a elegir para la investigación a parejas en las que ellas desempeñen un trabajo a tiempo completo, a fin de que el empleo sea central para ellas antes de ser madres, como lo es para sus compañeros varones, también empleados a tiempo completo. He considerado así la vida laboral de las mujeres como recurso central para que puedan negociar en pareja.

c) Los dos miembros de la pareja tienen estudios universitarios. Este es un elemento clave para mantener relaciones de género igualitarias (Castro y Pazos, 2008; Martín-García y Baizán, 2006; Esping-Andersen y Brodmann, 2005; Cowdery y Knudson-Martín, 2005; Himmelweit y Sigala, 2004). Cuando la participación laboral de las mujeres es mayor y mayor su nivel de estudios, más participación en el hogar habrá por parte de los varones, a su vez influidos por su mayor estatus y su nivel educativo más elevado (Meil, 1997).

d) Los dos miembros de la pareja tienen empleos no manuales y, para acceder a los mismos, han necesitado una titulación universitaria. El nivel educativo superior y el desempeño de un trabajo no manual, al que haber accedido gracias a una titulación universitaria, son fundamentales como criterios de selección muestral, por posibilitar mayor equilibrio de género en las parejas.

e) Parejas que han convivido al menos durante seis meses, considerado como el tiempo mínimo necesario para generar dinámicas de convivencia, aunque la mayoría lo han hecho a lo largo de más tiempo. Puede observarse el tiempo de convivencia de las parejas en la primera fase de entrevistas en la tabla 1.

f) Parejas que están esperando su primer descendiente y ni el varón ni la mujer han tenido hijos anteriormente. Es el primer hijo común el que influye en los usos de los tiempos (Bott, 1990), por tanto, he considerado fundamental que la experiencia de ser madre y padre suceda durante el proceso de investigación: «Si es difícil no ser ama de casa para una mujer soltera, para una casada es casi imposible [...] una vez que llegan los hijos, la conversión de la madre en ama de casa está consumada» (Durán, 1978: 19).

g) Ambos miembros tienen una posición social media. La homogeneidad socioeconómica es clave en la investigación, ya que permite extraer conclusiones sobre las dinámicas de género desarrolladas por estas parejas. He identificado el contexto socioeconómico a partir de su nivel de ingresos, del nivel de estudios y del empleo desempeñado. Esta homogeneidad posibilita que el

Tabla 1. Tiempo de convivencia de las parejas de la muestra en la primera entrevista, en meses

\begin{tabular}{ccccccccccccc}
\hline Ríos & Luna & Sierra & Lago & Pino & Valle & Flores & Sol & Castaño & Montes & Isla & Naranjo Arroyo \\
\hline 6 & 52 & 6 & 24 & 30 & 36 & 12 & 8 & 60 & 48 & 12 & 18 & 72 \\
\hline
\end{tabular}

Fuente: elaboración propia. 
Tabla 2. Edad de la mujer en la primera entrevista, en años cumplidos

\begin{tabular}{|c|c|c|c|c|c|c|c|c|c|c|c|c|}
\hline Ríos & Luna & Sierra & Lago & Pino & Valle & Flores & Sol & Castaño & Montes & Isla & Naranjo & Arroyo \\
\hline 34 & 31 & 39 & 30 & 28 & 35 & 31 & 36 & 32 & 34 & 36 & 36 & 38 \\
\hline
\end{tabular}

Fuente: elaboración propia.

análisis se centre en el género, considerado como categoría de análisis central en los contextos de investigación ${ }^{2}$.

La edad de cada miembro de la pareja, especialmente de la mujer, no se ha tenido en cuenta a priori como criterio de selección muestral, no obstante, posteriormente, se ha identificado como criterio con alta capacidad explicativa sobre las relaciones de género mantenidas. Así, de manera coherente con los criterios anteriores, si se selecciona a mujeres que desempeñen un empleo no manual a tiempo completo que sea central en sus vidas antes de ser madres, no podrán ser muy jóvenes, es por eso que las mujeres de la muestra tienen más de 30 años $^{3}$. Además, la edad se ha identificado como un recurso fundamental para una mayor igualdad en la pareja cuando ella es mayor que él. En la tabla 2, pueden observarse las edades de las mujeres durante las primeras entrevistas.

Los criterios anteriores de selección muestral, fundamentados teóricamente, y de acuerdo con la teoría de género y con estudios previos (véase el primer epígrafe), se han considerado los mínimos comunes que las parejas deben cumplir para ser incluidas en la muestra. El resto de características sociodemográficas específicas de cada pareja seleccionada, como el nivel socioeconómico de la familia de origen, el ámbito rural o urbano, el hecho de contar con ayuda familiar para los cuidados, el tiempo disponible para varones y mujeres al margen del empleo o el mantenimiento de un tiempo propio al margen de la pareja y del bebé, entre otros, se han tenido en cuenta en el análisis en función de su capacidad explicativa para comprender las relaciones de género de cada pareja en su contexto social específico.

\section{Por qué entrevistar por separado y no conjuntamente}

La aportación clave del artículo es el diseño de un estudio longitudinal a partir de entrevistas abiertas y por separado en dos momentos en el tiempo a dos personas que viven una misma realidad. Entrevistar a cada miembro de la pareja por separado facilita poder hablar sobre uno mismo y la propia vida

2. En el anexo 1, pueden observarse los estudios y la ocupación de las mujeres y de los varones de las parejas entrevistadas.

3. Todas, excepto una. Que la mujer tenga igual o mayor edad que el varón es un importante indicador de cambio social, además de funcionar como recurso relevante para la negociación en pareja y para la modificación de las relaciones de poder dentro de la misma. De hecho, no es lo más común entre la población en general, ya que, tradicionalmente, las mujeres se han emparejado con varones mayores que ellas, lo cual ha facilitado el mayor dominio masculino en la pareja. 
cotidiana con suficiente libertad y tranquilidad. Su ventaja es que no aumenta la censura estructural que existe en cualquier situación social, ya que no está el otro miembro de la pareja presente.

También las entrevistas conjuntas presentan ventajas, la principal es posibilitar la interacción discursiva, es decir, permite que se negocie el sentido de los discursos en el momento en el que son expresados y así reconstruir los relatos de forma más completa, además de ayudar a la memoria. No obstante, presentan una desventaja fundamental: el aumento de la censura estructural que ya de por sí está presente en cualquier interacción comunicativa, y el aumento de la autocensura, por estar el otro miembro de la pareja presente. Además, en las conjuntas, es más difícil tratar los temas conflictivos, los temores, los reproches o los asuntos que generan mayor malestar, aspectos clave para el objeto de investigación.

Las entrevistas por separado han posibilitado momentos de mucha intensidad y «sinceridad», lo cual permite realizar el análisis y la valoración del significado práctico de la maternidad y la paternidad, así como de las relaciones de pareja en la vida cotidiana. En la investigación, se han considerado prioritarias las bondades de las entrevistas por separado, por sus potencialidades para llegar a conocer las prácticas en torno a los cuidados y a las tareas domésticas. A su vez, ha conllevado un mayor número de entrevistas y ha complejizado el trabajo de campo y el análisis, lo cual ha enriquecido enormemente el objeto de investigación, ya que se dispone de cuatro entrevistas abiertas por cada pareja, mayor cantidad de información y de mayor calidad, con enorme potencialidad para el análisis. No obstante, las entrevistas realizadas a tres de las parejas fueron conjuntas. Se procedió así para poder contrastar metodológicamente el tipo de discursos que se pueden obtener con las entrevistas conjuntas frente a las efectuadas por separado.

Tras la realización de la investigación, puedo afirmar que, si se tiene la pretensión de comprender las diferentes prácticas en torno a la primera maternidad y paternidad en sus contextos cotidianos, las entrevistas por separado son más adecuadas, a pesar de sacrificar la interacción de la pareja en el momento de llevarlas a cabo. Puede pensarse que realizar entrevistas por separado pueda causar cierto recelo en la pareja, pero una vez que aceptan participar, lo entienden bien, ya que la visión sobre una misma realidad es diferente para cada uno de ellos. En las cuatro citas siguientes, puede apreciarse el tipo de discurso que se puede obtener en las entrevistas por separado.
¿Al final? Te digo yo, te lo digo pa que lo sepas, que, ¿el niño? Es pa tí... Es que, te llega a absorber tanto, que, de verdad, llegas a pensar, y adoras a tu niño, pero llegas a pensar que no tienes vida, que no tienes tiempo para tí. Es como decir: «Existo, o sea, yo estoy, existo, sigo siendo Marina». Porque ya tienes como una prolongación y no sabes muy bien lo que eres. (Marina Montes, técnica de administración pública, $2 .^{a}$ entrevista, por separado)

El sexo es uno de los temas que se mencionan en las entrevistas por separado, ausente en las entrevistas conjuntas. Por ejemplo, en la pareja Lago, él manifiesta la necesidad de aumentar la frecuencia de sus prácticas sexuales. 
Durante el embarazo, ha habido un tiempo que era muy bueno el sexo, de repente, de justo el primer tiempo, pues con la emoción, pues, tampoco lo he tenido yo mucho en la cabeza, pero después ha habido un momento en que yo he vuelto a, a tener muchas ganas de sexo, y Marina, no. Y también eso nos ha originao muchas tensiones... Al final, yo termino mencionándolo más veces de lo que a ella le apetecería, y pa mí sigue siendo insuficiente la frecuencia, y lo que hay... Si yo le cuento que, para mí es importante, que necesito más sexo, que una vez por semana, como mucho, me parece insuficiente, o lo que sea, las veces que sea, y ella, es que es impensable ahora mismo, es que, posiciones demasiao alejás, ya hasta que no podemos comprendernos, pues no se da un paso, pero, aún así, el problema sigue estando, ¿no? (Javier Lago, técnico en medio ambiente, $2 .^{\mathrm{a}}$ entrevista, por separado)

También la mayor necesidad de sexo del varón frente a la mujer está presente en la pareja Luna. Este tema es tratado por ambos en sus entrevistas por separado, y posiblemente no habría salido de haberse realizado una entrevista conjunta. Téngase en cuenta que el sexo no era un tema por el que se preguntara directamente, ya que no estaba presente en el guión de entrevista, sino que se ha tratado si la persona entrevistada lo ha considerado relevante y lo ha mencionado durante su relato.

¿Él? Necesita, que yo le dé mucho más, de lo que le doy, y ese es siempre, siempre, siempre, siempre, su lema: «Marina, no me das lo que yo necesito...». El sexo también es una cosa importante, que, tampoco le doy lo que él necesita. ¿Sabes?... Es, que él es más ardiente que yo, él es más, ¿̧sabes? Y yo soy más apagailla, ¿sabes? Y entonces, entre el sexo, y el, cariño. Ėl tiene ahí una falta. (Marina Luna, diseñadora de moda, $1 .^{\mathrm{a}}$ entrevista, por separado)

\section{Así expresa una mayor necesidad sexual Javier Luna:}

Es como parte de su, obligación de mujer, o del matrimonio, sabe que, ¿yo? Tengo esas necesidades, y ella intenta complacérmelas, ¿`sabes? Pueees, no seee. (Breve silencio.) Tema sexo. ¿Sabes? Ahora no tanto, ¿no? Pero, ella siempre ha sío más dejá... Yo siempre, he querio más sexo, y ella, no. Ella ha sío más tranquila. Pues, lo hemos hablao, y muchas veces, yo sé que ella, a lo mejor, sin apetecerle tanto, lo puede hacer por mí. Pos igual que el tema sexo, otras cosas de cariño, de estar más pendiente, de, de tener un detalle bonito, de ¿sabes? Pues ella, muchas veces, uhmm, se esfuerza en hacerlo. (Javier Luna, director de sucursal bancaria, $1 .^{\mathrm{a}}$ entrevista, por separado)

En las entrevistas conjuntas, no se hace referencia en ningún momento al sexo. Esto es muestra de la censura estructural que existe cuando está la pareja presente. Esta es una ausencia significativa, ya que surge en las entrevistas por separado, aunque no se pregunte explícitamente por él. Además, hay otros temas que no son tratados de estar la pareja presente. Si alguno de los temas conflictivos son tratados con los dos, aparece mucha autocensura, muchos miramientos, funciona constantemente la comunicación no verbal para tratar 
que la pareja no cuente demasiado, se palpa la tensión, y la información no fluye con la naturalidad que lo hace en las entrevistas por separado. En las entrevistas conjuntas se negocia constantemente el sentido de los discursos, cada miembro de la pareja trata así de mostrar una «buena cara» de sí mismo y, a la vez, intenta evitar ser juzgado por su pareja y por la persona que entrevista. Además, en las entrevistas conjuntas, es necesario tomar en consideración un texto más extenso para poder contextualizar la interacción negociada, como podemos observar en la siguiente interacción, cuya cita aparece a continuación, mientras que, en las entrevistas por separado, es posible encontrar núcleos de condensación de sentido ${ }^{4}$ breves y muy claros en un texto menos extenso, como muestran las citas anteriores.

ENTREVISTADORA: ¿Algún punto de vista diferente, sobre algún tema, aunque sea mínimo? (Silencio.)

Javier Castaño: Pues, no sé.

Marina CASTAÑo: Yo no he sío capaz de sacar ninguno. No sé, estoy pensando...

Javier CASTAÑo: No sé, porque habría una cosa, pero bueno, es que, no sé. Yo es que soy de la opinión de que, hasta que no estás en una situación real, no puedes decir, y hay una cosa, que, se planteó en su momento, y que, bueno, yo no sé si me va a llevar la contraria,

MARINA CASTAÑO: Estoy pensando.

JaVIER CASTAÑO: ¡Uy! ¡Ya me está mirando con caras raras!

MaRINA CASTAÑo: No, no te estoy mirando. Estoy pensando. Es que no sé por dónde vas a salir. No sé lo que vas a hablar.

Javier Castaño: No, no. Hubo un momento en el que, durante el embarazo, cuando, bueno, cuando te hablan de la prueba por el tema del síndrome de Down y eso.

Marina Castaño: Sí.

Javier Castaño: Pues bueno, pues te planteas: «Bueno, y si nos la hacemos y nos da positivo, y después te haces la amniocentesis, y también te da... ¿Qué hacemos? ¿Abortamos?». Entonces, yo creo, te digo por lo que hablamos, que Marina habría sido a lo mejor partidaria en su momento de abortar, cuando yo...

MARINA CASTAÑO: ¿Yo? ¡Si te dije que yo no me la quería hacer!

Javier CASTAÑo: ¿¿No? Pero, vamos a ver, pero, ¿si hubiera llegao el momento de que a tí te dan con seguridad de que pudiera tener un síndrome de Down...?

Marina CaStaño: ¡Hombre! Te dije que, a lo mejor, un síndrome de Down no. Ahora, si te dicen que vas a tener... Pero te dije, hombre, ya, yéndonos muy al extremo.

JAVIER CASTAÑo: jJoer, pero es que ya!

MARINA CASTAÑO: $¡$ Que te digan con seguridad que se va a quedar toa su vida en una silla de ruedas!

4. En terminología de Alfonso Ortí. Se refiere al texto nuclear sociológica y emocionalmente más sobrecargado de la entrevista, coherente con el resto de ella, para otorgar un sentido general a todo el discurso. 
Javier CASTAÑo: ¡Pero, vamos a ver! (Momento tenso.) Me he ido al extremo... Yo creo que, en ese tema, no sé, a lo mejor hubiéramos tenío, pareceres diferentes.

MARINa CASTAÑo: Pero fíjate que, al final, los dos dijimos: «iNada, palante!». JaVier CASTAÑo: ¡Noo! Por eso te he dicho que, sería al principio, porque yo sé que, luego, sin embargo, cuando ella ha estao embarazá, y ya lo ha sentío dentro y to eso, yo creo que, su forma de pensar ha cambiao. (Breve silencio.) ¿Sabes? Yo creo que ha cambiao, sin necesidad ni de que, también te digo, es una cosa, que hasta que no estás... Es muy bonito decir: «Yo no, yo no abortaría». Pero, hay que ponerse en la piel del que lo está sufriendo en ese momento y el que le pone en esa tesitura en ese momento.

Marina CASTAÑO: ¡Noo, pero lo habíamos pensao!

(Marina Castaño, profesora de universidad, y Javier Castaño, político, 1. ${ }^{\mathrm{a}}$ entrevista, conjunta)

Por todo ello, para el objetivo de investigación, las entrevistas por separado son más adecuadas que las conjuntas. Entrevistar a las mismas parejas en diferentes momentos de su ciclo vital es metodológicamente estratégico, precisamente para poder conocer las expectativas y las previsiones durante el embarazo y la experiencia y las prácticas en torno a la maternidad y la paternidad una vez que ya han tenido lugar. Todo sucede durante el transcurso de la investigación.

\section{Cómo un cuidado trabajo de campo vela por la calidad de la investigación}

Tanto el diseño de la investigación, como la selección muestral y la contactación de las parejas se han llevado a cabo en todo momento con esmero y cuidado. Con ello, se ha tratado de garantizar la validez del trabajo de campo $y$, por tanto, de la investigación. La calidad en el trabajo de campo significa evitar ejercer la violencia simbólica que puede estar presente en la investigación, huyendo de la imposición y optando por entrevistar a quienes, cumpliendo con los criterios teóricos de selección muestral, ofrecen buena disposición para participar y colaborar, con su historia y su discurso, con los objetivos pretendidos.

Las parejas han sido contactadas mediante redes sociales sin que tuvieran una relación previa con la persona que les entrevistó. Este tipo de contactación posibilita la existencia de cierta reciprocidad u obligación moral una vez que han aceptado participar en la iniciativa. A las parejas se les informaba que sus entrevistas contribuirían a llevar a cabo una investigación que trataría sobre las relaciones de pareja cuando se está esperando el primer bebé. A priori, puede parecer difícil conseguir la colaboración de las parejas. Ciertamente, no ha sido nada fácil. Si ha sido posible, se ha debido a que el trabajo de campo se ha prolongado durante cuatro años ${ }^{5}$, tiempo que ha permitido que la contactación diese sus frutos, puesto que ha sido necesario que la gestación llegara a su fin, que el bebé naciese y que pasara

5. La primera entrevista de la primera fase se realizó el día 21 de diciembre de 2006 y la última entrevista de la segunda fase, el día 20 de septiembre de 2010. 
un año aproximadamente para realizar las segundas entrevistas. La contactación es una fase complicada, ya que hay que informar a la pareja, pero no insistir ni tratar de convencerla. Cuidando este proceso, se garantiza el buen desarrollo del trabajo de campo y se vela así por la validez y la confiabilidad de la investigación.

La fase de contactación requiere una buena dosis de paciencia y empatía. Antes de contar con una pareja, es imprescindible que ambos miembros hayan consensuado su participación en las entrevistas. Una vez contactadas las parejas, se les llamaba por teléfono para informarles del objetivo general de la investigación, verificar que cumplen los criterios de selección muestral, obtener información adicional sobre la pareja y comprobar su disponibilidad para hablar de su vida cotidiana e implicarse en la investigación.

Ciertamente, no todas las parejas a las que se propuso participar accedieron. Se desestimaron aquellas que mostraban recelo o solo quería colaborar uno de los miembros. Se procedió de esta manera para prevenir la falta de franqueza y abandonos potenciales en la segunda fase; un riesgo que, de haberse producido, habría puesto en duda la validez de los resultados. La buena predisposición de las parejas ha permitido su sinceridad al tratar aspectos personales de su vida cotidiana, así como su fidelización para las entrevistas posteriores al nacimiento. La fidelización también se ha conseguido manteniendo un contacto periódico con los participantes en la investigación tras la primera fase.

Las entrevistas se han realizado en las viviendas de las parejas, lo que permite conocer su contexto vital, información que ha sido útil para el proceso de análisis. Tanto los nombres propios, como los de lugares que aparecían en los discursos se sustituyeron por seudónimos utilizados para salvaguardar la identidad de las personas entrevistadas sin alterar el sentido de lo que expresaron. Se utilizó una grabadora digital, lo cual garantizaba la nitidez de la grabación y la fidelidad de las entrevistas.

Como recomienda Bertaux (1993), las transcripciones se realizaron una vez finalizada cada entrevista. Asimismo, durante las mismas, se tomaron notas sobre su desarrollo para facilitar su comprensión y su análisis posterior. Las transcripciones fueron revisadas y repasadas junto a su audición para asegurar que respondían fielmente al discurso grabado. La primera lectura sobre papel también se realizó a la vez que se oía la grabación. Para la presentación de resultados, a todas las mujeres se les llamó con el mismo nombre ficticio, «Marina», igual que a todos los varones, "Javier». Al mismo tiempo, se utilizó el mismo apellido ficticio para cada pareja, lo que facilita su identificación y su relación en cada cita (véase la tabla 3).

Tabla 3. Nombres y apellidos ficticios de las parejas de la muestra

1. ${ }^{\text {a: }}$ Marina y Javier Ríos. 2. ${ }^{\text {a: }}$ Marina y Javier Luna. 3. ${ }^{\text {a: }}$ Marina y Javier Sierra.

4. ${ }^{\text {a: }}$ Marina y Javier Lago. 5. a $^{\text {a }}$ Marina y Javier Pino. 6. ${ }^{\text {a: }}$ Marina y Javier Valle.

7. ${ }^{\text {a: }}$ Marina y Javier Flores. 8. ${ }^{\text {a. }}$ Marina y Javier Sol. 9. a.: Marina y Javier Castaño.

10. a: Marina y Javier Montes. 11. a.: Marina y Javier Isla. 12. a. Marina y Javier Naranjo.

13. a: Marina y Javier Arroyo.

Fuente: elaboración propia. 
Tabla 4. Ejemplos de algunos temas del guión y sus temas de análisis correspondientes

\begin{tabular}{cc}
\hline \multicolumn{2}{c}{ Relaciones en la familia de origen $\left(1 .^{a}\right.$ entrevista) } \\
Algunos temas del guión & Temas de análisis \\
\hline
\end{tabular}

Vida cotidiana en la familia de origen.

Relaciones con padre y madre.

Relaciones con hermanos y hermanas.

Relaciones de la familia con el exterior (familia extensa, amistades, etc.).

Cambios en la familia (número de miembros, cambio de residencia, ciudad, etc.).
Entorno sociofamiliar en familia de origen.

Procesos de socialización diferenciada por razón de género entre hermanos y hermanas, relaciones de autoridad, procedencia social. Capital social de la familia de origen.

\section{Relaciones externas a la familia de origen en la adolescencia y en la juventud Relaciones con amigos y amigas (1. ${ }^{a}$ entrevista)}

Algunos temas del guión

Temas de análisis

Expectativas y aspiraciones familiares. Formar Valor de la maternidad y la paternidad, además una familia, tener hijos, etc. de la formación de una familia como expectativa desde la juventud o como nueva cuestión.

\section{Relaciones con la pareja actual (1. ${ }^{\mathrm{a}}$ y $2 .^{\mathrm{a}}$ entrevistas)}

Algunos temas del guión

Temas de análisis

Decisiones que se toman en la pareja en torno Estrategias de interacción y proceso de toma de a cuestiones centrales (coche, casa, hipoteca, lugar de residencia, préstamos, dinero, gestión de la economía familiar, boda, etc.). decisiones en la pareja. Conflictos, consensos.

Conflictos implícitos, temas y momentos que generan malestar, cuestiones no resueltas.

Valorar quien dirige la negociación según los temas o los ámbitos. Centralidad y prioridades de cada miembro de la pareja.

Costes de las decisiones adoptadas, renuncias, malestares, acuerdos implícitos.

Otros elementos que entran en juego en las negociaciones, como la intromisión de la familia de origen.

\section{Relación de pareja (2. ${ }^{a}$ entrevista)}

Algunos temas del guión

Decisiones adoptadas (nombre del bebé, lac- Proceso de toma de decisiones, participación tancia a demanda o con horario, pañales, leche en la toma de decisiones, cómo deciden, cómo materna u otra alimentación, etc.).

discuten o llegan a acuerdos, sobre qué temas concretos hablan, prioridades.

Temas de conflicto: frecuencia y forma como discuten sobre temas de actualidad, relaciones con la familia, cuestiones de dinero, cuidado y atención del bebé, aspectos relacionados con la educación de la criatura, sobre el tiempo que pasan juntos y temas de pareja en general.

Procesos vividos de continuidad o ruptura de la pareja. Prioridades y puntos de inflexión por los que uno de los miembros no está dispuesto a ceder. Procesos de negociación en la práctica.

Fuente: elaboración propia. 
Para la realización de las entrevistas, se diseñaron dos guiones temáticos: uno para las entrevistas llevadas a cabo durante el embarazo y otro para las llevadas a cabo un año después del nacimiento del bebé. Cada guión se ha derivado del marco teórico y se ha perfilado tras entrevistar en cada fase a las primeras parejas de la muestra. Cada entrevista, al ser abierta, ha seguido su propio proceso discursivo, aunque en todas ellas se han tratado los temas previstos en el guión.

La introducción con la que comenzaron las entrevistas de la primera fase, las realizadas durante el embarazo, fue la siguiente. Se trató que fuese sencilla y coloquial, así como que no obviase ninguna de las cuestiones de las que, a priori, se debe informar a cualquier sujeto que participe en una investigación (que el tratamiento de la información será anónimo, que la entrevista va a ser grabada, que se pedirá su consentimiento), así como el objetivo general de la investigación. Debido a que se había reservado previamente al menos una hora y media con cada miembro de la pareja, no se consideró necesario volverlo a resaltar en la introducción.

Introducción utilizada al comienzo de las primeras entrevistas: «Estoy realizando una investigación sobre las parejas y sus relaciones cotidianas antes y después de la llegada de su primer bebé. Sería interesante conocer vuestra experiencia. Si no te importa, me gustaría grabarla para poder atender mejor a lo que hablemos y no tener que tomar notas continuamente. Todo lo que me cuentes es confidencial y la información que se utilice se hará garantizando el anonimato".

Cada uno de los temas previstos en el guión, que estaba dividido en diferentes bloques temáticos, se ha pensado en relación con los posibles análisis que podía poner en juego. En las entrevistas realizadas durante el embarazo, se han tratado las relaciones en la familia de origen, las relaciones externas a la familia de origen en la adolescencia y juventud y las relaciones con la pareja actual. En las entrevistas realizadas cuando el bebé tenía un año, se trata lo acontecido tras el nacimiento para cada madre y cada padre, en relación con su pareja y con su entorno social. En la tabla 4, se puede apreciar, para cada bloque temático, la relación entre algunos de los temas del guión y los temas de análisis vinculados a estos.

\section{Cómo el proceso de análisis contribuye a la validez}

Para lograr el objetivo de investigación y velar por la validez de la misma, he considerado apropiado y recomiendo seguir dos tipos de análisis específicos y complementarios: un análisis de discurso y un análisis desde la perspectiva de género, además de establecer un sistema de categorías de análisis saturadas.

El análisis de discurso realizado se entiende como práctica social contextualizada, generada a partir de unas condiciones determinadas de producción que le dotan de sentido social. Los discursos no se conciben con un sentido verdadero, sino como prácticas en sí mismas con sentido para quienes los emiten. He seguido el siguiente procedimiento metodológico para analizar los discursos: 
exploración exhaustiva de los textos, producción analítica de los esquemas de interpretación - lógica en uso- y fundamentación de las interpretaciones comparando, como recomienda Martín Criado (1995). Todos los discursos tienen una dimensión ideológica presente en todo sistema significante cuyo sentido está determinado socialmente. Así, el análisis de discurso debe permitir una descripción de campo de efectos de sentido y de sus condiciones de producción. De la misma manera, por partir de unas condiciones de producción y de reconocimiento determinadas, tienen una dimensión ideológica (Verón, 2004) contemplada en el análisis.

Continuando con esta idea, Cicourel (1989) plantea la importancia de los contextos para analizar los procesos de negociación, así, en el proceso de análisis, el sentido ha sido puesto en relación con el contexto social en el que se ha generado cada uno de los discursos presentes en las entrevistas. De este modo, he tenido en cuenta tanto las situaciones informales como las formales de las parejas analizadas. De hecho, Cicourel otorga mucha relevancia a los contextos informales por la importancia de las relaciones previas en la configuración de las relaciones sociales posteriores, por más formalizado que sea el contexto de partida. Por esta razón, en las entrevistas se habla sobre la historia social y económica de cada miembro y su interpretación sobre el proceso seguido por la pareja, para, así, poder interpretar el presente de las parejas a partir de su pasado.

El análisis realizado da más importancia a las prácticas sobre las ideas para resolver lo cotidiano, algo que coincide con los hallazgos de investigaciones feministas que muestran que las vidas de las mujeres siguen sobre todo una acción responsable, más que una acción intencional (Lengermann y NiebruggeBrantley, 1993). Es decir, siguen más lo incidental que lo intencional o racional, puesto que la acción depende de las mujeres, por ejemplo, del momento del ciclo vital en el que se encuentran o de las respuestas que pueden dar para satisfacer las demandas y las necesidades propias y de sus familias. El enfoque anterior es coherente con el planteado por Martín Criado (2004), cuando cuestiona que la acción se derive de los contenidos mentales, como plantea la teoría parsoniana de la acción. Así, el análisis se centra fundamentalmente en las prácticas sociales cotidianas y en la forma como los sujetos sociales se adaptan a su entorno y resuelven su vida, tomando decisiones estratégicas que dependen de los contextos en los que actúan. «Prácticas y discursos ponen en juego tensiones: entre las distintas normas o legitimidades que pesan sobre las prácticas, entre lo que se piensa que se debería hacer y lo que se hace - que se aleja siempre de lo que se percibe como legítimo» (Martín Criado, 2010: 350). Son los problemas cotidianos los que deben resolverse en la práctica diaria, lo que depende de los recursos con los que se cuente, que se pueda y se decida movilizar. El análisis conecta, así, con el enfoque teórico de Norbert Elias (1982) y su concepto de poder coyuntural y situacional, que es posible modificar mediante la movilización de recursos entre quienes interaccionan, idea que se desarrolla al principio de este artículo.

El análisis ha tenido en cuenta los criterios teóricos de selección muestral de manera fundamental por ofrecer el contexto desde el que interpretar los 
discursos de cada mujer y cada varón, antes y posteriormente a su maternidad y paternidad. Así, el análisis de los discursos ha partido de los contextos que los han generado, de las características sociodemográficas de las parejas y del momento del ciclo vital en que se encontraban. He analizado el sentido de cada entrevista, no como un sentido continuado concatenado y lógico, sino como un sentido social, con coherencia en una situación social concreta, bajo unas condiciones determinadas de producción y en el marco de una censura estructural determinada (Martín Criado, 1991). En cualquier caso, el eje de análisis principal se ha centrado en las dinámicas sociales, en las condiciones de posibilidad, en los elementos empíricos derivados de la experiencia sobre las categorías analizadas, con atención especial a las prácticas que favorecen o dificultan la existencia de relaciones más igualitarias entre géneros. He analizado las estrategias puestas en juego por cada miembro de la pareja en interacción con el otro miembro y con su entorno, en los contextos sociales investigados.

Paralelamente, he seguido también un análisis de género, sin despojarlo de su razón de ser feminista, entendido el género como categoría política forjada por el feminismo, como «ideología transformadora de la realidad» (Cobo, 2005: 257). Este análisis supone una opción por un enfoque capaz de luchar contra las desigualdades de género y de otra índole, ya que las inequidades condicionan el acceso y el control a recursos y beneficios, así como a las opciones vitales, ya sean éstas tiempo social, empleo, trabajo, dinero, salud, expectativas sociales o de la propia existencia. No obstante, considerar el género como categoría de análisis central no significa considerarla siempre como categoría de análisis principal en todos los contextos y situaciones analizadas (Breilh, 1994). En concreto, el análisis de género del objeto de investigación ha partido de las herramientas analíticas que pueden observarse en la tabla 5.

El análisis se centra en la realidad discursiva. Esto no significa dejar de lado el nivel material de los procesos sociales, entendidos como condiciones sociales de vida, realidad laboral, familiar y personal y las expectativas de

Tabla 5. Herramientas utilizadas para el análisis de género

a) Análisis de las relaciones de influencia entre los géneros.

b) Análisis del trabajo remunerado y del no remunerado, o de gestión del hogar y de cuidados.

c) Análisis de la segregación en los entornos familiares.

d) Análisis social y real de los tiempos vividos. Análisis del uso y reparto de los tiempos entre ambos géneros.

e) Análisis de la división sexual / de género del trabajo.

f) Análisis del acceso y el control a recursos y beneficios.

g) Análisis de la satisfacción de las necesidades prácticas, simultáneamente o no a la consecución de intereses estratégicos.

h) Participación en la toma de decisiones. Análisis sobre cómo se toman las decisiones estratégicas en las parejas, concretamente, entre cada miembro, respecto a oportunidades personales, laborales, familiares $\mathrm{y}$, en definitiva, vitales.

i) Análisis de las estrategias de interacción y negociación.

Fuente: elaboración propia. 
Tabla 6. Sistema de categorías de análisis definitivo

1. Nueva paternidad o paternidad más igualitaria.

1.1. Con relación al empleo.

1.2. Con relación a los cuidados.

1.3. Con relación al uso de los tiempos.

1.4. Dinámicas de pervivencia del modelo de padre tradicional.

1.5. Contradicciones identificadas.

2. Nueva maternidad o maternidad más igualitaria.

2.1. Con relación al empleo.

2.2. Con relación a los cuidados.

2.3. Con relación a los usos del tiempo.

2.4. Dinámicas de pervivencia del modelo tradicional de la «buena madre».

2.5. Contradicciones identificadas.

3. Nuevas relaciones de pareja más igualitarias.

3.1. Interacciones, negociaciones, resolución de conflictos, toma de decisiones.

3.2. Organización posterior al nacimiento para permitir la conciliación entre los ámbitos del trabajo, la familia y la vida personal.

3.3. Consecuencias no previstas de las decisiones y de las acciones adoptadas.

3.4. Dinámicas de pervivencia de las parejas de roles tradicionales.

3.5. Contradicciones identificadas.

Fuente: elaboración propia.

cambio de las mismas, ya que es en referencia a estos procesos como cada género construye su forma de dar sentido a su existencia social. En la investigación, se ha valorado la importancia de los ámbitos de trabajo remunerado, no remunerado y de tiempo libre, analizando en qué medida cada ámbito articula a los demás.

Complementariamente al análisis de discurso y de género, he establecido un sistema de categorías de análisis clave derivadas y construidas a partir del marco teórico y de la conceptualización y contextualización del objeto de estudio. El sistema de categorías se diseñó en función de su relevancia y de su sentido, y no por su mayor frecuencia a lo largo de los discursos. He relacionado el contenido atribuido a las representaciones del mundo de la vida partiendo del acuerdo y del posicionamiento de cada persona entrevistada con las categorías y las dimensiones de análisis (Lozares et al., 1998). Las categorías han sido también analizadas de forma estructural, es decir, habiendo probado los principios que organizan los elementos del discurso, respondiendo a los objetivos de analizar las ideologías, las representaciones y las aspiraciones, así como su transformación. Y, a su vez, he analizado también las estrategias puestas en juego, las situaciones conflictivas, los componentes de una situación problemática, las interpretaciones de un acontecimiento, las reacciones latentes o la reconstitución de las realidades pasadas (Quivy y Campenhoudt, 1998: 218). Las categorías de análisis se consideran los conceptos clave que ha interesado tratar de forma teórica.

El sistema de categorías finalmente adoptado ha partido de diferentes clasificaciones iniciales, que se han ido probando en los análisis realizados a las entrevistas llevadas a cabo en ambas fases a las primeras parejas de la muestra. 
El sistema de categorías con el que se inició el análisis era mucho más extenso y exhaustivo, tanto que realmente no era posible encontrar un nivel de saturación aceptable para todas las categorías. El sistema finalmente adoptado (véase la tabla 6) comprende las categorías saturadas clave y ofrece la sencillez necesaria sin sacrificar en exhaustividad, por ello se ha mostrado como el más adecuado para el análisis del objeto de investigación. Las categorías finalmente adoptadas no son mutuamente excluyentes, son categorías de sentido, ya que los discursos abiertos están cargados de sentidos y significados y pueden referirse a distintos aspectos en función de donde se quiera poner el acento.

Este último sistema de categorías contribuye al objetivo de investigación, identificando las dinámicas sociales, estructurales, sociodemográficas y prácticas que fomentan, de estar presentes, la equidad de género. Además, y de manera más relevante, las dinámicas, las acciones y los comportamientos que permiten la agencia por parte de las madres y los padres para constituirse en la práctica en «nuevas» madres y "nuevos» padres $^{6}$, para formar parte de parejas más igualitarias alejadas del desempeño de roles tradicionales segregados para cada género.

\section{Debatiendo en torno a la calidad y a la validez de las propuestas metodológicas presentadas}

Los apartados metodológicos presentados se sostienen porque fundamentan la consecución de una investigación válida y de calidad. Así, la validez es un concepto fundamental en la investigación social, ya que indica si lo que nos proponemos con la investigación, así como su metodología y la forma de interpretar los datos, son apropiados — válidos - para explicar y comprender el objeto de investigación. El problema de la validez comenzó a plantearse en ciencias sociales, fundamentalmente con relación al método cuantitativo; no obstante, la validez también es fundamental en la investigación cualitativa, aunque se plantea en un sentido distinto a los conceptos clásicos de validez y fiabilidad, ya que éstos están relacionados, en su surgimiento, evolución y aplicación, con la investigación cuantitativa y, concretamente, con la encuesta.

Esto no quiere decir que, para el método cualitativo, no haya otros criterios que velen por su validez, y, más concretamente en esta investigación, que indiquen que el método, la técnica de investigación y el proceso y las decisiones metodológicas adoptadas son adecuados y válidos para lo que teóricamente se pretendía. Del mismo modo, la validez de la investigación permite afirmar que las prácticas, las dinámicas y las estrategias de interacción analizadas son «verdaderas", "coherentes lógicamente» y que han sido conocidas como realmente están funcionando en los contextos sociales de estudio.

6. La nueva madre es la madre con un empleo central en su vida que incorpora el trabajo de cuidados y gestión del hogar, pero no de forma exclusiva; y el nuevo padre, además de ser proveedor, es un padre disponible y responsable de los cuidados (Dermott, 2006) y de las tareas domésticas. El desarrollo teórico sobre nueva maternidad y nueva paternidad será objeto de una publicación posterior. 
Para evitar el sesgo de corrección política de las entrevistas, se ha procedido en las mismas con confianza, incluso ingenuidad, insistiendo en aquella información que ha presentado dudas, pero sin mostrar la incorrección o la inconsistencia directamente a la persona entrevistada. Para conocer la información sobre las interacciones, las negociaciones y los conflictos, no he preguntado directamente por ésta, sino que he tratado situaciones concretas que han ido surgiendo a lo largo del relato, tratando y concretando aspectos muy específicos a partir de ejemplos concretos. Se ha preguntado qué pasó, cómo lo resolvieron, qué hicieron, así como si hubo distintos puntos de vista o diferentes formas de resolver las interacciones y los conflictos. Tomar los discursos como prácticas en sí mismas resuelve la cuestión de la corrección política, ya que se analizan los discursos sobre las acciones, las prácticas y los comportamientos, que es de lo que realmente se dispone, no las acciones en sí mismas, ya que para eso deberían haberse podido observar tal y como sucedieron en la vida cotidiana.

Además, para velar por la validez de la investigación, he tratado de garantizar un nivel de saturación de los discursos aceptable. Con ello, he acreditado que la realidad de las estrategias de interacción y negociación, así como las dinámicas mostradas, sean las que realmente son, dentro de las posibilidades reales que ofrece la investigación en ciencias sociales. Respecto al criterio de saturación, se puede afirmar haber llegado a un número óptimo de sujetos en la muestra, cuando nuevas entrevistas no ofrecen información adicional sustancialmente diferente y relevante (Bertaux, 1993). La saturación discursiva es el principal argumento que ofrece el método cualitativo de investigación para garantizar la validez y contrarrestar las críticas que afirman que la investigación cualitativa carece de ella. Han sido muchos los temas tratados en las entrevistas y no puedo afirmar haber conseguido un nivel de saturación en todos ellos, pero sí en los que fundamentan los análisis realizados ${ }^{7}$. La homogeneidad de la muestra contribuye a la consecución de la saturación de las categorías, por tanto, es fundamental para avalar la validez de la investigación. La homogeneidad, además, mejora la confiabilidad y validez de la muestra (Franklin y Ballan, 2001), lo cual permite contar con una de menor tamaño.

En la práctica, la limitación de recursos económicos y temporales impide muchas veces ser tan ambiciosos respecto a la saturación como lo fueron la pareja compuesta por Daniel Bertaux e Isabelle Bertaux-Wiame (1993), que entrevistaron a panaderos de toda Francia durante diez años y llegaron a realizar un centenar de historias de vida. No obstante, alcanzar el nivel de saturación debe ser el objetivo. En esta investigación, ha sido un criterio pragmático de aproximación, más que una prueba irrefutable de la validez externa de la misma. Además, se ha tratado que sea una investigación válida, tanto por acceder a las estructuras o a los procesos objetivos, como por acceder a los esquemas simbólicos o procesos subjetivos, como recomienda Bertaux (1993).

7. Los resultados de la investigación cuya metodología se discute en el presente artículo serán objeto de una publicación posterior. 
La validez externa es una cuestión criticada a la investigación cualitativa, pero su garantía puede fundamentarse con relación a la captación, o lo que en este artículo hemos nombrado "contactación», y por la forma como se diseña el análisis de discurso. Con ello, se permite generalizar los análisis a partir de las condiciones en que se produce el discurso (Martín Criado, 1997). Así, para avalar la validez son, por tanto, claves el diseño muestral y la forma como se ha hecho la contactación, así como conseguir la saturación de los discursos en las categorías analizadas.

Siguiendo con el problema de la validez de la investigación cualitativa, Ruiz de Olabuenaga (1996) apela a la confiabilidad como criterio de excelencia, concepto que incluye cuatro criterios de calidad relacionados entre sí que los datos cualitativos deberían reunir: credibilidad o valor de verdad, transferibilidad o aplicabilidad, dependencia o consistencia y confirmabilidad respecto al problema de la neutralidad. Además, debido a las críticas que argumentan que esto no resuelve el problema de la validez en la investigación cualitativa, el mismo autor, basándose en Guba, propone exigir y asegurar ciertos criterios o controles metodológicos para garantizar la excelencia en la investigación cualitativa mediante la confiabilidad (Guba, en Ruiz de Olabuenaga, 1996: 109).

El diseño metodológico presentado ha tratado de cumplir estos controles. Así, he contrastado las interpretaciones realizadas a partir de los datos con diferentes investigadores, tratando, a su vez, de romper con los sesgos de sentido común iniciales que, como sujetos sociales, tenemos al investigar, además de apoyar siempre los análisis en los datos. De la misma manera, con la construcción del marco teórico, he documentado los constructos analíticos utilizados y los criterios de selección muestral, con lo cual he retroalimentado teoría y datos en busca de la significación teórica de las interpretaciones, y he tratado, en general, de cumplir con los criterios de confiabilidad.

\section{Conclusiones y discusión}

El objetivo del presente artículo ha sido mostrar cómo he procedido en la práctica para explicar las dinámicas sociales que pueden favorecer o dificultar una mayor igualdad de género en parejas heterosexuales no tradicionales durante el primer año de vida de su primer bebé. Es decir, mostrar las decisiones metodológicas sobre el diseño de investigación llevado a cabo y los principios teóricos que las fundamentan y, a la vez, recomendar aspectos metodológicos concretos para investigar y comprender la forma como se forjan las relaciones cotidianas y, específicamente, de poder (en la acepción de Elias, 1982) en parejas que tratan de alejarse de la tradicionalidad en el desempeño de sus roles y responsabilidades.

El enfoque cualitativo ha permitido acercarse, de manera longitudinal y comprensiva, a las mujeres y a los varones entrevistados en su transición y durante su primera maternidad y su primera paternidad. Un momento de alta demanda de tiempo para cuidar que suele afianzar las diferencias de género 
en parejas que, durante la convivencia sin hijos, sí habían logrado un mayor equilibrio de género en sus interacciones cotidianas y en las responsabilidades asumidas.

Creo haber justificado las razones por las que he elegido, dentro del método cualitativo de investigación, la entrevista abierta como la técnica de estudio más adecuada. Esta permite acercarse a los esquemas simbólicos de los sujetos sociales que interaccionan, así como a los procesos objetivos y prácticos que enmarcan las interacciones cotidianas en torno a los cuidados y a las tareas domésticas, pero también respecto al empleo y a las relaciones sociales. Este proceso metodológico permite conocer las contradicciones y las transformaciones que se están produciendo cuando operan diferentes legitimidades en parejas alejadas del modelo tradicional que, no obstante, mantienen algunas prácticas vinculadas a este modelo junto a prácticas más igualitarias.

He justificado teóricamente los criterios de selección muestral adoptados, así como la homogeneidad de la muestra, para poder explicar las dinámicas sociales que fomentan, si están presentes, la igualdad de género. Además de justificar la bondad de realizar entrevistas por separado en lugar de conjuntas, si se pretende evitar una mayor censura estructural de los discursos y ayudar a que sean tratados en las entrevistas los temas más conflictivos, los temores, los reproches o los asuntos que generan malestar, además de aspectos concretos sobre los diferentes procesos de negociación en los que se implica cada miembro de la pareja. He especificado también el proceso de análisis seguido, que se basa tanto en un análisis de discurso, como en un análisis desde la perspectiva de género, y he construido además un sistema de categorías saturadas clave que fundamentan la consistencia de los resultados.

En su parte final, el artículo argumenta la validez y la calidad de la investigación cualitativa longitudinal llevada a cabo a partir de los conceptos de homogeneidad de la muestra, de saturación de las categorías de análisis y de confiabilidad de la investigación realizada. Considero necesario seguir abordando objetos de investigación micro, como las relaciones de pareja y la vida cotidiana, desde una mirada cualitativa para comprender cómo tienen lugar las prácticas en los contextos sociales que las generan. Investigaciones realizadas bajo el mismo marco metodológico pueden ser útiles para ofrecer información relevante que ayude a tomar decisiones en relación con las políticas públicas de empleo y cuidados, ya que un ámbito no puede ser entendido sin el otro, así como para otras políticas sociales que puedan fomentar la corresponsabilidad de mujeres y varones, si se pretenden promover relaciones de género más equilibradas. 


\section{Referencias bibliográficas}

Alonso Benito, Luis Enrique (1998). La mirada cualitativa en sociología: Una aproximación interpretativa. Madrid: Fundamentos.

BABCOCK, Linda y LASCHEVER, Sara (2003). Women don't ask: Negotiation and the gender divide. Oxford: Princeton University Press.

BECKER, Gary (1981). "Altruism in the family and selfishness in the market place». Economica, 48, 1-15.

Beltrán, Miguel (1998). «Cinco vías de acceso a la realidad social». En: García FERrAndo, Manuel; IBÁÑEZ, Jesús y Alvira, Francisco (comp.). El análisis de la realidad social: Métodos y técnicas de investigación. Madrid: Alianza, 17-45. (Universidad Textos)

BERTAUX, Daniel (1993). «La perspectiva biográfica: validez metodológica y potencialidades». En: MARINAS Javier Miguel y SANTAMARINA, Cristina (eds.). La historia oral: métodos y experiencias. Barcelona: Debate, 149-230.

Bertaux, Daniel y Bertaux-Wiame, Isabelle (1993). «Historia de vida del oficio de panadero». En: MARINAS, Javier Miguel y SANTAMARINA, Cristina (eds.). La historia oral: métodos y experiencias. Barcelona: Debate, 231-250.

Botía, Carmen (2010). «Negociar en la vida cotidiana para transformar las relaciones de género: una propuesta teórica». Papers: Revista de Sociologia, 95 (1), 119-137.

Вотт, Elisabeth (1990). Familia y red social: Roles, normas y relaciones externas en las familias urbanas corrientes. Madrid: Taurus Humanidades.

BreIlH, Jaime (1994). Género, poder y salud. Quito: Ibarra Ediciones, CEAS-UTM.

BRUllet, Cristina (1996). «Prácticas de crianza e identidades parentales». En: CARRASQUER OTO, Pilar y BRUlleT TREnAS, Cristina (coord.). Sociología de las relaciones de género: Congreso Sociología, Granada, 1995. Madrid: MTAS. Instituto de la Mujer, 45-66.

CARABAÑA, Julio (1993). «Educación y estrategias familiares de reproducción». En: GiL Calvo, Enrique y Garrido, Luis. Estrategias familiares. Madrid: Alianza Editorial, 37-47.

CARrasco, Cristina et al. (2003). Malabaristas de la vida: Mujeres, tiempos y trabajos. Barcelona: Icaria. (Más Madera)

Castro, Carmen y Pazos, María (2008). «Permiso de maternidad, de paternidad y parentales en Europa: Algunos elementos para el análisis de la situación actual». En: PAzos, María (dir.). Economía e igualdad de género: Retos de la Hacienda Pública en el siglo XXI. Madrid: Instituto de Estudios Fiscales, 185-222. (Estudios de Hacienda Pública)

CiCOUREL, Aaron (1989). «Texto y contexto: aspectos cognitivos, lingüísticos y organizativos de la investigación de campo en las negociaciones internacionales». Papers: Revista de Sociologia, 31, 33-58.

Cово, Rosa (2005). "El género en las ciencias sociales». Cuadernos de Trabajo Social, $18,249-258$.

CONDE, Fernando (2008). "Los grupos triangulares como "espacios transicionales" para la producción discursiva: Un estudio sobre la vivienda en Huelva». En: GORDO, Ángel J. y SERRANO, Araceli. Estrategias y prácticas cualitativas de investigación social. Madrid: Pearson Educación.

CONNELl, Raewyn W. (1987). Gender and power: Society, the person and sexual politics. Stanford: Stanford University Press.

Coria, Clara (1996). La negociación nuestra de cada día. Buenos Aires: Paidós. 
Cowdery, Randi S. y Knudson-Martin, Carmen (2005). "The construction of motherhood: Tasks, relational connection, and gender equality». Family Relations, $54,335-345$.

Dema Moreno, Sandra (2005). «Entre la tradición y la modernidad: las parejas españolas de doble ingreso». Papers: Revista de Sociologia, 77, 135-155.

- (2006). Una pareja, dos salarios: El dinero y las relaciones de poder en las parejas de doble ingreso. Madrid: CIS.

DERMOTT, Esther (2006). «What's parenthood got to do with it?: men's hours of paid work». The British Journal of Sociology, 57 (4), 619-634.

- (2008). Intimate Fatherhood: A sociological analysis. Londres: Routledge.

Durán, María Ángeles (1978). El ama de casa: Crítica política de la economía doméstica. Bilbao: Zero Zyx, 87. (Lee y Discute)

Durán, María-Ángeles y RogERO GARCÍA, Jesús (2009). La investigación sobre el uso del tiempo. Madrid: CIS.

ELIAS, Norbert (1982). Sociología fundamental. Barcelona: Gedisa.

EsPing-Andersen, Gosta y BrodmanN, Stefanie (2005). "When mathers work and fathers care: Joint household fertility decisions in Denmark and Spain». Working Papers 5 Demosoc. Barcelona: Universitat Pompeu Fabra.

FrankLin, Cynthia S. y BALLAN, Michelle (2001). "Reliability and validity in qualitative research». En: THYER, Bruce A. The handbook of social work research methods. Thousand Oaks, California: Sage, 273-292.

Glaser, Barney G. y STRAuss, Anselm L. (1967). The Discovery of Grounded Theory: Strategies for Qualitative Research. Chicago: Aldine Publishing Company.

HaKim, Catherine (2000). Work-Lifestyle Choices in the 21 $1^{\text {st }}$ Century: Preference Theory. Oxford: Oxford University Press.

HARTMAnN, Heidi (1981). "The family as the locus of gender, class, and political struggle: The example of housework». Signs: Journal of Women in Culture and Society. University of Chicago, 3 (6), 366-394.

Himmelweit, Susan y Sigala, Maria (2004). "Choice and the relationship between identities and behaviour for mothers with pre-school children: Some implications for policy from a UK study». Journal of Social Policy, 33 (3), julio, 455-478.

Lengermann, Patricia Madoo y Niebrugge-Brantley, Jill (1993). «Teoría feminista contemporánea». En: RitZER, George. Teoría sociológica contemporánea. Madrid: McGraw-Hill, Interamericana de España, 353-409.

Lozares, Carlos; Carrasquer, Pilar y Domínguez, Marius (1998). «Las representaciones en el mundo de la vida cotidiana». Papers: Revista de Sociologia, 55, 131-149.

Manser, Marilyn y Brown, Murray (1980). "Marriage and household decisionmaking: A bargaining analysis». International Economic Review, 21 (1), 31-44.

MARTín CRIADO, Enrique (1991). "Del sentido como producción: Elementos para un análisis sociológico del discurso». En: LATIESA, Margarita (ed.). El pluralismo metodológico en la investigación social: Ensayos típicos. Granada: Servicio de Publicaciones de la Universidad de Granada, 187-212.

- (1995). «Propuestas de método para evitar la deriva interpretativa en la producción y análisis sociológico de discursos». Comunicación presentada en el V Congreso Español de Sociología, 28-30 de septiembre. Granada: Federación Española de Sociología.

- (1997). «El grupo de discusión como situación social». REIS: Revista Española de Investigaciones Sociológicas, 79, 81-112.

- (2004). «El valor de la buena madre: Oficio de ama de casa, alimentación y salud entre las mujeres de clases populares». Revista Española de Sociología, 4, 93-118. 
- (2010). «Las tallas grandes perjudican seriamente la salud: La frágil legitimidad de las prácticas de adelgazamiento entre las madres de clases populares». Revista Internacional de Sociología, 68 (2), 349-373.

MARTÍN-GARCÍA, Teresa y BAIZÁN, Pau (2006). «The impact of the type of education and of educational enrolment on first births». European Sociological Review, 22, 259-275.

MeIL, Gerardo (1997). «La participación masculina en el cuidado de los hijos en la nueva familia urbana española». Papers: Revista de Sociologia, 53, 77-99.

- (2011). «El uso de los permisos paternales por los hombres y su implicación en el cuidado de los niños en Europa». Revista Latina de Sociología, 1, 61-97.

Miller, Tina (2007). «Is this motherhood is all about?: Weaving experiences and discourses through transition to first/time motherhood». Gender \& Society, 21, 337-358.

- (2011). «Falling back into Gender? Men's narratives and practices around firsttime fatherhood». Sociology, 45, 1094- 1109.

Murillo, Soledad (1996). El mito de la vida privada: De la entrega al tiempo propio. Madrid: Siglo XXI.

PATTON, Michael Quinn (1990). Qualitative evaluation and research methods. Newbury Park, California: Sage.

POLE, Christopher y LAMPARD, Richard (2002). Qualitative and quantitative methods in social research. Essex: Pearson Education.

Quivy, Raymond y CAMPENHOUdT, Luc Van (1998). Manual de investigación en Ciencias Sociales. México D.F.: Limusa, Noriega Editores.

Regnier-Loilier, Arnaud (2009). "Does the birth a child change the division of household tasks between partners?». Population \& Societies, 461, 1-4. Institut National d'Études Démographiques.

Risman, Barbara J. (1998). Gender Vertigo: American Families in Transition. New Haven: Yale University Press.

- (2004). «Gender as a social structure: Theory wrestling with activism». Gender \& Society, 18 (4), agosto, 429-450.

Ruíz de OlabuenaGa, José Ignacio (1996). Metodología de la investigación cualitativa. Bilbao: Universidad de Deusto.

SEgalen, Martine (1992). Antropología histórica de la familia. Madrid: Taurus.

TAYLOR, Steven J. y BogdAN, Robert (2002). "La entrevista en profundidad». En: TAYLOR, Steven J. y BOGDAN, Robert. Introducción a los métodos cualitativos de investigación. Madrid: Paidós, 100-132.

TORNS, Teresa (2008). «El trabajo y el cuidado: Cuestiones teórico-metodológicas desde la perspectiva de género». Empiria: Revista de Metodología de Ciencias Sociales, 15, enero-junio, 53-73.

VERÓN, Eliseo (2004). La semiosis social: Fragmentos de una teoría de la discursividad. Barcelona: Gedisa.

Weismann, Stephanie et al. (2008). «Not worth mentioning: The implicit and explicit nature of decision-making about the division of paid and domestic work». Community, Work and Family, 11 (4), noviembre, 341-363. Routledge.

ZuO, Jiping y BIAN, Yanjie (2001). "Gendered resources, division oh housework, and perceived fairness - A case in urban China». Journal of Marriage and Family, 63, 1122-1133. 
Anexo 1. Ocupaciones y niveles de estudios de los varones y las mujeres de las parejas de la muestra

\begin{tabular}{|c|c|}
\hline Padres y madres & Nivel de estudios y ocupaciones \\
\hline 1. a Javier Ríos & $\begin{array}{l}\text { Ingeniero agrícola. Funcionario grupo A y jefe de servicio } \\
\text { de administración autonómica. }\end{array}$ \\
\hline 1. ${ }^{\text {a Marina Ríos }}$ & Licenciada en Derecho. Administrativa de administración autonómica. \\
\hline 2. ${ }^{\text {a Javier Luna }}$ & Licenciado en Economía. Director de sucursal bancaria. \\
\hline 2. ${ }^{\mathrm{a}}$ Marina Luna & $\begin{array}{l}\text { Diseñadora de moda. Modista. FP II. Es la única madre de la muestra } \\
\text { sin estudios universitarios, lo que se ha tenido en cuenta en el análisis. }\end{array}$ \\
\hline 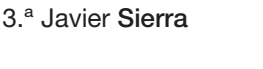 & $\begin{array}{l}\text { Licenciado en Ciencias Políticas. Mando intermedio en una fundación } \\
\text { pública. }\end{array}$ \\
\hline 3. ${ }^{\mathrm{a}}$ Marina Sierra & $\begin{array}{l}\text { Licenciada en Traducción e Interpretación. Intérprete en la Unión Euro- } \\
\text { pea. }\end{array}$ \\
\hline 4. ${ }^{\text {a Javier Lago }}$ & $\begin{array}{l}\text { Licenciado en Ciencias Ambientales. Técnico en medio ambiente } \\
\text { de empresa pública. }\end{array}$ \\
\hline 4. ${ }^{\text {a }}$ Marina Lago & Licenciada en Arquitectura. Autónoma con estudio propio. \\
\hline 5. ${ }^{\text {a Javier Pino }}$ & Licenciado en Musicología. Profesor de enseñanza secundaria. \\
\hline 5. ${ }^{a}$ Marina Pino & $\begin{array}{l}\text { Diplomada en Graduado Social. Jefa de administración en empresa } \\
\text { privada de construcción. }\end{array}$ \\
\hline 6. ${ }^{\text {a Javier Valle }}$ & $\begin{array}{l}\text { Licenciado en Economía. Contable en empresa de distribución de pro- } \\
\text { ductos dietéticos. }\end{array}$ \\
\hline 6. ${ }^{a}$ Marina Valle & $\begin{array}{l}\text { Diplomada en Magisterio y licenciada en Antropología. } \\
\text { Técnica de ayuntamiento. }\end{array}$ \\
\hline 7. ${ }^{\text {a Javier Flores }}$ & $\begin{array}{l}\text { Licenciado en Veterinaria. Veterinario autónomo para asociación } \\
\text { de ganaderos. }\end{array}$ \\
\hline 7. ${ }^{a}$ Marina Flores & $\begin{array}{l}\text { Licenciada en Psicopedagogía y Logopedia. Pedagoga y logopeda } \\
\text { infantil autónoma. }\end{array}$ \\
\hline 8. a Javier Sol & $\begin{array}{l}\text { Licenciado en Periodismo. Propietario de una pequeña empresa } \\
\text { de producción de espectáculos y conciertos. }\end{array}$ \\
\hline 8. ${ }^{\mathrm{a}}$ Marina Sol & Licenciada en Bellas Artes. Artista. \\
\hline 9. ${ }^{\text {a Javier Castaño }}$ & Licenciado en Derecho. Político. \\
\hline 9. ${ }^{\mathrm{a}}$ Marina Castaño & Licenciada en Empresariales. Profesora de universidad. \\
\hline 10. ${ }^{\mathrm{a}}$ Javier Montes & Diplomado en Magisterio. Maestro infantil. \\
\hline 10. ${ }^{a}$ Marina Montes & $\begin{array}{l}\text { Licenciada en Derecho. Técnica de promoción de empresas de mujeres } \\
\text { en una empresa pública. }\end{array}$ \\
\hline $11 .^{a}$ Javier Isla & $\begin{array}{l}\text { Licenciado en Empresariales. Gerente y propietario de empresa familiar } \\
\text { de importación e inversiones con países del Este. }\end{array}$ \\
\hline 11. ${ }^{\text {a }}$ Marina Isla & $\begin{array}{l}\text { Licenciada en Economía. Directora de sucursal bancaria para } \\
\text { empresas. }\end{array}$ \\
\hline 12. ${ }^{\text {a Javier Naranjo }}$ & Licenciado en Farmacia. Propietario y encargado de farmacia familiar. \\
\hline 12. ${ }^{\text {a }}$ Marina Naranjo & Diplomada en Magisterio. Maestra infantil. \\
\hline 13. ${ }^{a}$ Javier Arroyo & $\begin{array}{l}\text { Licenciado en Ciencias Físicas. Beca contrato tesis doctoral y formador } \\
\text { FPO. }\end{array}$ \\
\hline 13. ${ }^{a}$ Marina Arroyo & $\begin{array}{l}\text { Diplomada en Graduada Social. Responsable de administración } \\
\text { de un sindicato. }\end{array}$ \\
\hline
\end{tabular}

